\title{
CAVI, a New Parameter That Detects Arterial Stiffness Change After Smoking
}

\author{
Yoichiro Hirata, MD; Masataka Sata, MD, PhD
}

$\mathbf{C}$ igarette smoking is a well-known cardiovascular risk factor, which deteriorates both coronary and peripheral circulation. Smoking causes vascular damage, resulting in endothelial dysfunction and harmful hemodynamic effects, such as increased arterial stiffness. ${ }^{1}$ Besides smoking, a number of factors and conditions, such as diabetes, dyslipidemia, hypertension, renal dysfunction, obesity and chronic obstructive pulmonary disease potentially influence arterial stiffness. Smoking interacts with these factors and increases arterial stiffness and cardiovascular risk. To predict and prevent cardiovascular event, it is important to establish standard physiological parameters and/or biomarkers to detect acute and chronic vascular damages caused by risk factors.

\section{Article p 698}

Many clinical studies have been performed to determine the effect of acute, chronic and passive smoking on arterial stiffness. ${ }^{2}$ Acute smoking was found to cause an acute increase in arterial stiffness. Kim et al reported that the brachial-ankle pulse wave velocity (baPWV) was significantly higher in chronic smokers $5 \mathrm{~min}$ after cigarette smoking compared to non-smokers and remained higher for $30 \mathrm{~min}$, although the baPWV at baseline was not significantly different between the 2 groups. ${ }^{3}$ However, Rhee et al reported that smoking induced no significant increase in heart-femoral PWV after adjustment for total cholesterol, time-dependent heart rate and brachial mean arterial pressure. ${ }^{4}$ There are also many studies that investigated the effects of chronic smoking on PWV. ${ }^{2}$ The majority of studies identified chronic smoking as a risk factor for increasing arterial stiffness. ${ }^{5}$ It was reported that brachial-radial PWV was significantly increased in smokers. Nguyen et al reported that cigarette smoking was found to be a significant predictor of increased aortic PWV in white and black individuals. ${ }^{6}$ Similarly, passive smoking increased arterial stiffness acutely and chronically. However, some studies found no statistical difference in arterial stiffness between non-smokers and long-term smokers, although chronic smoking seems to sensitize the arterial response to acute smoking. Yufu et al found no significant difference between smokers and non-smokers when evaluating baPWV. ${ }^{7}$ In addition, some other groups found no significant correlation between smoking status and increased carotid-femoral PWV. ${ }^{8}$ Taken together, the role of chronic smoking in increasing arterial stiffness is still slightly controversial. Moreover, it remained unclear whether arterial stiffness is reversed after smoking cessation. ${ }^{2}$ The effect of smoking discontinuation on arterial stiffness remains to be established by prospective smoking cessation trials using a standard physiological parameter to assess arterial stiffness. ${ }^{2}$

Stiffness of large arteries has been related to cardiovascular mortality, but the mechanisms underlying this relationship have not been fully understood. One of the most common analyses to estimate arterial stiffness is PWV. ${ }^{9}$ Several different methods to measure PWV have been shown to be useful for diagnosing arteriosclerosis. However, PWV measurement has been reported to be influenced by several factors such as blood pressure (BP), autonomic nerve function, and therefore does not reflect arteriosclerosis in some cases. ${ }^{10}$

Recently, cardio-ankle vascular index (CAVI) was developed to evaluate the stiffness of the aorta, femoral artery and tibial artery non-invasively. ${ }^{11}$ CAVI is adjusted for BP based on the stiffness parameter beta and should measure arterial stiffness independent of BP. ${ }^{12}$ It was demonstrated that CAVI is a reproducible index of arterial distensibility, which is not influenced by BP changes during measurement. ${ }^{12}$ Izuhara et al reported that high CAVI implies progression of carotid and coronary arteriosclerosis and that CAVI is more closely linked with arteriosclerosis than PWV. ${ }^{13}$ Kadota et al also reported the availability of CAVI as a screening tool for atherosclerosis. ${ }^{14}$

In this issue of the Journal, Kubozono et al elegantly demonstrated that smoking causes a significant increase in arterial stiffnesss as measured by baPWV and CAVI. ${ }^{15}$ They analyzed 10 male smokers to evaluate the acute effect of smoking on arterial stiffness. A total of 160 male active smokers were assessed to evaluate the chronic effect. In the acute study, baPWV and CAVI increased immediately after smoking. In the chronic study, baPWV and CAVI were significantly correlated with mean BP and the Brinkman index. In multiple regression analysis, baPWV was independently correlated with mean BP. However, CAVI was independently correlated with the Brinkman index, but not with mean BP. Receiver operating characteristics (ROC) curves of baPWV and CAVI to predict the Brinkman index $\geq 500$ demonstrated that area under the ROC curve of CAVI was higher than that of baPWV. Based on these findings, the authors concluded

The opinions expressed in this article are not necessarily those of the editors or of the Japanese Circulation Society.

Received January 5, 2011; accepted January 5, 2011; released online January 27, 2011

Department of Cardiovascular Medicine, Institute of Health Biosciences, the University of Tokushima Graduate School, Tokushima

(Y.H., M.S.); Department of Pediatrics, University of Tokyo Graduate School of Medicine, Tokyo (Y.H.), Japan

Mailing address: Masataka Sata, MD, PhD, Department of Cardiovascular Medicine, Institute of Health Biosciences, the University of

Tokushima Graduate School, 3-18-15 Kuramoto-cho, Tokushima 770-8503, Japan. E-mail: sata@clin.med.tokushima-u.ac.jp

ISSN-1346-9843 doi:10.1253/circj.CJ-10-1319

All rights are reserved to the Japanese Circulation Society. For permissions, please e-mail: cj@j-circ.or.jp 
that smoking causes a significant increase of arterial stiffness as measured by baPWV and CAVI. The authors suggest that CAVI might be superior than baPWV to evaluate the degree of arterial stiffness caused by smoking.

Kubozono et al's findings along with recent literatures suggest that CAVI might show promise to be a valuable parameter to evaluate arterial stiffness influenced by smoking. CAVI could be helpful to estimate therapeutic efficacies of several interventions on lifestyle-related diseases. Clinical utilities of CAVI remain to be validated by multi-center prospective studies.

\section{References}

1. Celermajer DS, Adams MR, Clarkson P, Robinson J, McCredie R, Donald A, et al. Passive smoking and impaired endotheliumdependent arterial dilatation in healthy young adults. $N$ Engl J Med 1996; 334: 150-154.

2. Doonan RJ, Hausvater A, Scallan C, Mikhailidis DP, Pilote L, Daskalopoulou SS. The effect of smoking on arterial stiffness. Hypertens Res 2010; 33: 398-410.

3. Kim JW, Park CG, Hong SJ, Park SM, Rha SW, Seo HS, et al. Acute and chronic effects of cigarette smoking on arterial stiffness. Blood Press 2005; 14: 80-85.

4. Rhee MY, Na SH, Kim YK, Lee MM, Kim HY. Acute effects of cigarette smoking on arterial stiffness and blood pressure in male smokers with hypertension. Am J Hypertens 2007; 20: 637-641.

5. Levenson J, Simon AC, Cambien FA, Beretti C. Cigarette smoking and hypertension: Factors independently associated with blood hyperviscosity and arterial rigidity. Arteriosclerosis 1987; 7: 572-577.
6. Nguyen QM, Srinivasan SR, Xu JH, Chen W, Berenson GS. Racial (black-white) divergence in the association between adiponectin and arterial stiffness in asymptomatic young adults: The Bogalusa heart study. Am J Hypertens 2008; 21: 553-557.

7. Yufu K, Takahashi N, Hara M, Saikawa T, Yoshimatsu H. Measurement of the brachial-ankle pulse wave velocity and flow-mediated dilatation in young, healthy smokers. Hypertens Res 2007; 30: $607-$ 612.

8. Rehill N, Beck CR, Yeo KR, Yeo WW. The effect of chronic tobacco smoking on arterial stiffness. Br J Clin Pharmacol 2006; 61: $767-773$.

9. Meguro T, Nagatomo Y, Nagae A, Seki C, Kondou N, Shibata M, et al. Elevated arterial stiffness evaluated by brachial-ankle pulse wave velocity is deleterious for the prognosis of patients with heart failure. Circ J 2009; 73: 673-680.

10. Takaki A, Ogawa H, Wakeyama T, Iwami T, Kimura M, Hadano Y, et al. Cardio-ankle vascular index is a new noninvasive parameter of arterial stiffness. Circ J 2007; 71: 1710-1714.

11. Shirai K, Utino J, Otsuka K, Takata M. A novel blood pressureindependent arterial wall stiffness parameter; cardio-ankle vascular index (CAVI). J Atheroscler Thromb 2006; 13: 101-107.

12. Kubozono T, Miyata M, Ueyama K, Nagaki A, Otsuji Y, Kusano K, et al. Clinical significance and reproducibility of new arterial distensibility index. Circ J 2007; 71: 89-94.

13. Izuhara M, Shioji K, Kadota S, Baba O, Takeuchi Y, Uegaito T, et al. Relationship of cardio-ankle vascular index (CAVI) to carotid and coronary arteriosclerosis. Circ J 2008; 72: 1762-1767.

14. Kadota K, Takamura N, Aoyagi K, Yamasaki H, Usa T, Nakazato $\mathrm{M}$, et al. Availability of cardio-ankle vascular index (CAVI) as a screening tool for atherosclerosis. Circ J 2008; 72: 304-308.

15. Kubozono T, Miyata M, Ueyama K, Hamasaki S, Kusano K, Kubozono $\mathrm{O}$, et al. Acute and chronic effests of smoking on arterial stiffness. Circ J 2011; 75: 698-702. 